\title{
El valor del lugar en las relaciones de inclusión y exclusión en un colegio rural agrupado. Un estudio etnográfico
}

\author{
Space and relations of exclusion and inclusion \\ in a cluster school
}

\author{
Emilio Matías Solanilla*, Begoña Vigo Arrazola**
}

Recibido: 3 de abril de 2020 Aceptado: 18 de mayo de 2020 Publicado: 31 de julio de 2020

To cite this article: Matías Solanilla, E. y Vigo Arrazola, B. (2020). El valor del lugar en las relaciones de inclusión y exclusión en un colegio rural agrupado. Un estudio etnográfico. Márgenes, Revista de Educación de la Universidad de Málaga, 1 (2), 90-106 DOI: https://doi.org/10.24310/mgnmar.v1i2.8457

\section{RESUMEN}

En un contexto marcado por la transformación neoliberal de los servicios educativos y cuando la inclusión se presenta como un valor de referencia para el desarrollo sostenible, la finalidad de este artículo es contribuir a la comprensión del papel activo que juegan los lugares y las interacciones que se generan en ellos para la formación de experiencias de segregación o de inclusión en el entorno de la escuela rural. El objetivo es identificar cuáles son las experiencias de inclusión o exclusión entre los miembros de un Colegio Rural Agrupado (CRA). El estudio etnográfico realizado en una escuela adscrita a un CRA de la Comunidad de Aragón (España), a través de la observación participante, conversaciones informales y análisis de documentos, muestra cómo las interacciones generadas en torno a estructuras como la escuela y el CRA difieren respecto a las relaciones de proximidad entre las familias y el alumnado de una parte y los docentes de otra. Finalmente, se discuten las tensiones en relación con las experiencias vividas en las escuelas rurales, subrayando la necesidad de que las políticas educativas atiendan a las particularidades de las distintas escuelas.

Palabras clave: percepción del espacio; relación comunidad-escuela; escuela rural; análisis cualitativo

\section{ABSTRACT}

In a context marked by the neoliberal transformation of educational services and when inclusion is presented as a value for a sustainable development, this article aims to contribute to understanding the active role that places and interactions in them have by generating experiences of segregation or inclusion in rural spaces. The goal is to identify which are the experiences of inclusion and segregation among the members of a Grouped Rural School. The ethnographic study of a school in a Grouped Rural School from Aragón (Spain), through participant observation, informal conversations and document analysis, shows how the interactions generated around structures such as the school and the CRA differ on one hand to proximity relationships between families and students and on the other hand to the teachers. Finally, the tensions in relation to the experiences lived in rural schools are discussed, underlining the need for educational policies to attend to the particularities of the different schools.

Keywords: space perception; school-community relationship; rural school ; qualitative analysis

“Emilio Matías Solanilla 0000-0003-4976-0804

Educación. Gobierno de Aragón (España)

101069@unizar.es
**Begoña Vigo Arrazola 0000-0001-9734-8596

Universidad de Zaragoza (España)

mbvigo@unizar.es 


\section{N V E S T I G A C I Ó N}

\section{INTRODUCCIÓN}

Cuando la inclusión se presenta como un valor de referencia en el contexto actual atendiendo al desarrollo sostenible de los espacios que se encuentran en situación de desigualdad (UNESCO, 2018), la escuela rural constituye un tema crítico. La investigación en diferentes países subraya la situación de desigualdad del mundo rural y sus escuelas frente a las áreas urbanas, atendiendo a la prestación de servicios, a las posibilidades de elección, a la representación negativa que durante décadas les ha sido atribuida, y al escaso conocimiento de las mismas (Åberg-Bengtsson, 2009; Corbett, 2015; Llevot y Garreta, 2008; Ortega, 1995).

La fuerte despoblación sufrida en zonas rurales de Europa y especialmente de España, junto a las connotaciones negativas, como baja calidad o peores resultados que su homónima urbana, atribuidas con carácter externo, han servido para explicar numerosos cierres de escuelas pequeñas desde finales de los 50 (Hernández, 2000; Santamaría, 2012; Vigo y Soriano, en prensa). Para compensar esta situación de desventaja se han creado en distintos momentos diferentes alternativas organizativas hasta la consideración actual del Colegio Rural Agrupado (CRA). No obstante, siempre ha estado presente un modelo de escuela urbana estandarizada que ignoraba el contexto de referencia (Ortega, 1995; Tapia y Castro, 2014).

La situación de desigualdad y segregación experimentada por las escuelas rurales (Ortega, 1995; Tapia y Castro, 2014), en aumento en un contexto de mercado (Öhrn y Beach, 2019), junto al escaso interés por investigar, conocer y mejorar el funcionamiento de la escuela rural (Hargreaves, 2017) justifica el sentido de profundizar en su conocimiento a partir de las voces de los implicados.

La finalidad de este artículo es contribuir al conocimiento y a la mejora del funcionamiento de la escuela rural como servicio público en el contexto global actual (Camarero, 2017; Martínez y Bustos, 2011; Vázquez, 2016), tomando como referencia las voces de familias, alumnado y profesorado. El propósito es explorar los discursos y experiencias que tienen lugar en las escuelas rurales desde un marco teórico que subraya el papel activo que juegan los lugares y las interacciones que se generan en ellos para la formación de experiencias de segregación o de inclusión. Más concretamente, el objetivo es identificar y comprender las distintas experiencias e interpretaciones de familias, docentes y alumnado, en relación con su escuela y con el Colegio Rural Agrupado (CRA) al que pertenecen. Las preguntas de investigación que guían el estudio son las siguientes:

¿Cómo actúan y qué dicen las familias, docentes y alumnado de sus escuelas y de los colegios rurales agrupados propuestos por la administración educativa?

¿Cómo son descritas y experimentadas las relaciones de inclusión o exclusión por parte de docentes y familias de las escuelas rurales?

Para ello se toma como referencia un estudio etnográfico llevado a cabo en una escuela rural pequeña adscrita a un CRA de la Comunidad de Aragón.

\section{MARCO CONCEPTUAL, DE INVESTIGACIÓN Y TEÓRICO}

Las agrupaciones escolares rurales, también son conocidas como "cluster" o federación de escuelas (Giordano, 2008; Hargreaves, 2017). Ante el cierre y el aislamiento de las escuelas uni- 


\section{N V E S T I G A C I Ó N}

tarias, constituyen un medio para dar respuesta a la escolarización del alumnado residente en áreas rurales, para generar redes de colaboración y para optimizar la gestión en sus escuelas (Autti y Hyry-Beihammer, 2014).

España no ha sido ajena a esta tendencia (Corchón, Raso e Hinojo, 2013; Domingo, 2013) que, dependiendo de las comunidades autónomas, se ha presentado con denominaciones y características específicas (Boix, 2004). El colegio rural agrupado (CRA) consiste en la unión administrativa de las escuelas de varias localidades que llevan a cabo un proyecto educativo compartido (Abós y Lorenzo, 2019). Tiene la consideración de centro docente único con plena capacidad académica y de gestión, por lo que cuenta con los mismos órganos que cualquier colegio (Ponce de León, Bravo y Torroba, 2000). Se interpreta como un modelo que ha permitido mantener viva la escuela rural, garantizando la formación del alumnado en su entorno, mejorando los recursos humanos de los centros y mitigando el aislamiento de los maestros (Domingo, 2013). Pese a ello, tiene sus limitaciones desde la óptica de la reducción del número de escuelas (Vigo y Soriano, en prensa), el intento por asemejar su estructura a una escuela urbana con 'largos pasillos' (Ortega, 1995; Tapia y Castro, 2014), y la dificultad de los y las docentes para implicar a la comunidad de las distintas localidades que lo integran en un proyecto común (Ponce de León et al., 2000).

A la vista de la literatura sobre el tema de escuela rural, las interacciones entre escuela y comunidad presentan importantes variaciones de unas escuelas a otras y dentro de las mismas escuelas. Las interacciones entre docentes, alumnado y familias difieren significativamente dependiendo de los antecedentes de clase y de la ubicación social del hogar (Öhrn y Beach, 2019; Farrugia, 2020; Garreta, 2017). En ocasiones, los profesores marginan la relación con algunos grupos de población (Corbett, 2015; Yull, Wilson, Murray y Parham, 2018). Otras veces son determinadas familias quienes marginan la relación con la escuela dependiendo de su situación social y económica. En muchas localidades que han experimentado un incremento de la población desde el punto de vista residencial y laboral, la relación con la escuela es distinta entre los nuevos residentes y aquellas personas que cuentan con más arraigo en la localidad (Bagley y Hillyard, 2015; Johansson, 2017).

Distintos estudios ponen en valor las estrategias que tanto equipos directivos (Bagley y Hillyard, 2015; Lorenzo et al. 2019) como docentes (Álvarez y Vejo, 2017; Corbett, 2015; García y Pozuelos, 2017; Kalaoja y Pietarinen, 2009) llevan a cabo en las escuelas rurales para conseguir una relación fluida entre escuela y comunidad. Este hecho se presenta en una doble dirección. De una parte, cuando los docentes reconocen e implican a la comunidad en la vida de la escuela (Torres y Simovskca, 2017; Vigo y Soriano, 2015) y, de otra, cuando la escuela se implica en la vida de la comunidad (Bhengu y Miende, 2015; Sales, Moliner y Lozano, 2017), especialmente si estos docentes residen en el entorno y perduran en el centro (Morales, 2016). A la vista de los estudios revisados sobre el tema, estos se centran en la investigación de escuelas rurales independientes. Sin embargo, son escasas las investigaciones que indagan sobre las voces y vivencias de familias, docentes y alumnado, implicados en colegios rurales agrupados, atendiendo a las interacciones y las tensiones que se generan en un espacio rural concreto y en un contexto de mercado que sigue amenazando a estas escuelas. 


\section{N V E S T I G A C I Ó N}

$\mathrm{Al}$ análisis de investigaciones tomado como referencia en este artículo se añade como marco teórico el análisis de Massey (2012) respecto a los conceptos de espacio y lugar, de Soja (2014) en torno a la justicia espacial, y de Putnam (2002) a propósito del capital social.

Massey (2012) interpreta el espacio como un producto social, dotado de una dimensión de multiplicidad, en permanente construcción y formado por las prácticas y las interacciones que se dan tanto en nuestra vida íntima como de forma global. Asimismo, considera los lugares como momentos articulados en redes de relaciones e interpretaciones sociales donde una gran parte de las mismas están construidas a una escala mucho mayor que la que se define para ese momento como el lugar en sí mismo. Esto introduce una noción de lugar dinámica, sin fronteras y carente de una identidad única que no niega su unicidad como lugar. Según Soja (2014), el espacio es un elemento generador de desigualdades que, desde una perspectiva crítica, requiere un desarrollo de conocimiento y conciencia que posibilite un cambio del mundo a mejor. Siguiendo a Putnam (2002), el capital social que surge de las redes y las pautas de reciprocidad que se dan en las relaciones, tanto formales como informales, entre los miembros de una sociedad pueden generar mecanismos de cooperación y facilitar (o perjudicar) tanto el desarrollo individual como el de la propia sociedad.

Todo lo señalado hasta aquí nos lleva a una concepción de escuela como una realidad que no es estática ni tampoco delimitada territorialmente. No todas las escuelas rurales son iguales (Vigo y Beach, 2018) y múltiples actores, próximos o muy distantes físicamente con respecto a ellas (Poirey, 2006), influyen en su presente y futuro sometiéndolas a un permanente cambio. Igualmente, resulta de interés la no identificación que hace Massey (2012) entre lugar y comunidad al indicar que una comunidad puede existir en varios lugares y en un lugar puede haber varias comunidades.

En este marco conceptual, de investigación y teórico, surge la necesidad de ampliar información y profundizar en el conocimiento detallado de las voces de familias, docentes y alumnado con relación a las experiencias de inclusión o exclusión en su escuela y en su colegio rural agrupado.

\section{METODOLOGÍA}

Este artículo surge en un marco de investigación vinculado a dos proyectos I+D. El primero, en 2008, desde un modelo de investigación "ex post facto", se trata de un estudio descriptivo con un diseño no experimental transeccional basado en encuestación y grupos de discusión que investiga la atención a la diversidad en los núcleos rurales dispersos. El segundo proyecto, entre 2012 y 2016, basado en un diseño etnográfico, estudia la participación de las familias en los centros educativos de primaria y secundaria. El estudio que se presenta en este artículo constituye un ejemplo de análisis en profundidad en una escuela rural. El estudio se ha llevado a cabo mediante un diseño etnográfico (Hammersley y Atkinson, 2007) para el que se contó con el consentimiento de los miembros de la comunidad educativa implicados en el mismo. Los datos se recogieron a través de la observación (Hammersley, 2018), conversaciones informales (Spradley, 1979) a lo largo de 110 horas entre septiembre y junio del curso 2015/2016 y el análisis de documentos (Jachyra, Atkinson y Washiya, 2015). El trabajo de campo se ajustó al modelo intermitente planteado por Jeffrey y Troman (2004). Se consideró que era el diseño más adecuado 


\section{N V E S T I G A C I Ó N}

para conocer los comportamientos naturales de los miembros de la comunidad educativa y para comprender sus vivencias a partir de la participación del investigador como un miembro más de la misma, concretamente asumiendo el papel de profesor de apoyo.

El escenario de investigación es la Comunidad de Aragón (España), una comunidad en la que más del 50\% de la población se concentra en el núcleo urbano de la capital de la Comunidad y donde el número de CRAs y escuelas pequeñas con unidades que agrupan alumnado de distintos cursos constituye el 30\% de los centros educativos de Educación Primaria, según datos del curso 2014/2015 del Instituto Aragonés de Estadística. Más concretamente, el estudio que se presenta se desarrolla en una de estas escuelas, seleccionada en el contexto de los proyectos mencionados como un ejemplo representativo de las distintas voces que tienen lugar en una escuela rural. La escuela identificada con el pseudónimo de "Pueblogrande" forma parte de un Colegio Rural Agrupado llamado “Camporegadío”. Este CRA se constituyó en el curso 1996/97 con aulas en nueve localidades. La disminución del número de residentes ha provocado el desplazamiento del alumnado a otras poblaciones y un progresivo cierre de escuelas. Actualmente, solo hay 4 abiertas, sumando 8 aulas, 61 alumnos y 12 docentes, 5 de ellos itinerantes.

La escuela de Pueblogrande centraliza las tareas de gestión y administración del CRA. Es una localidad que según la Ley 45/2007, para el desarrollo sostenible del medio rural, es una zona rural a revitalizar. Su población es de 550 habitantes y como otras localidades del CRA se caracteriza por su baja natalidad y alto grado de envejecimiento. Sus residentes han nacido o trabajan en Pueblogrande, predominando la actividad agro-ganadera. Los efectos del desarrollo industrial primero y del mercado en la actualidad, han promovido el desplazamiento de su población a la ciudad desde los años 60 .

En el momento del estudio, la escuela investigada cuenta con 25 estudiantes, 20 familias y 7 docentes.

- El alumnado se encuentra distribuido en tres grupos multigrado. Un grupo incluye alumnado de Educación Infantil (3 niñas y 4 niños), otro incluye alumnado de los tres primeros cursos de Educación Primaria (2 chicas y 5 chicos) y el tercero, de los tres últimos cursos de Educación Primaria (5 alumnas y 6 alumnos).

- Las familias que llevan a sus hijos a la escuela, excepto una que reside en una localidad próxima, viven en Pueblogrande. Trece de estas familias cuentan con algín progenitor originario de Pueblogrande, cinco son parejas mixtas (español/a con extranjero/a) y en tres de ellas los dos cónyuges han nacido fuera de España.

- Los docentes son tres hombres y cuatro mujeres. Tres son funcionarios de carrera con destino en el CRA y cuatro son contratados interinos hasta final de curso. De estos, cinco son itinerantes y viajan de una escuela a otra. Los otros dos están fijos en la escuela como tutores de grupo. En el transcurso del trabajo de campo uno de ellos sufrió un percance y fue sustituido durante mes y medio por un maestro contratado. Para el profesorado interino, este curso es su primer año en esta escuela y sus expectativas para el próximo año son trabajar en otras escuelas más cercanas a su localidad de residencia. El profesorado funcionario de carrera lleva varios años trabajando en Pueblogrande y 


\section{N V E S T I G A C I Ó N}

no tienen intención de marcharse. Uno es el director, que cuenta con más de veinte años de destino en el centro y reside en un pueblo próximo. Otro es una maestra que lleva tres años en esta escuela, más de diez de experiencia laboral y reside en Pueblogrande. Estos son los únicos docentes que viven en la zona. El tercer funcionario lleva más de 10 años trabajados como docente y este es su cuarto curso en Pueblogrande. Este, de igual forma que el profesorado interino, vive en la capital de la provincia y se desplaza cada día desde su lugar de residencia, ajeno a su entorno de trabajo.

\subsection{Procedimiento}

El estudio de campo fue realizado por uno de los investigadores. Las primeras sesiones sirvieron de toma de contacto con el entorno y los protagonistas. El proceso inicial de la investigación se desarrolló entre septiembre y diciembre de 2015, tomándose notas desde los primeros días (Raggl, 2018). Se estimó necesario construir una comprensión holística de lo que ocurría en la escuela para organizar conceptualmente las ideas a lo largo de la investigación. Lejos de recoger los datos y llevarlos a casa para interpretarlos, el trabajo de campo se hizo despacio y sin perder de vista la relación con los estudios sobre el tema. Esta perspectiva etnográfica permitía comprender y aprender acerca de las experiencias que tenían lugar en la escuela (Beach, 2017). Al mismo tiempo se leían otros estudios etnográficos que ayudaron a contrastar y comprender lo que pasaba en el campo. Entre enero y abril de 2016 tuvo lugar el período más intenso de participación en la vida del centro y de recogida de la información. Los meses de mayo y junio de 2016 sirvieron para contrastar la información y extraer argumentos que explicaban lo que ocurría en la escuela y preparar la salida del campo que ya había sido negociada previamente. Después de nueve meses en la escuela, el material recogido fue analizado de nuevo y posteriormente devuelto al campo.

La observación participante se desarrolló durante todo el proceso de permanencia en el campo. La información era recogida por parte del investigador directamente del escenario natural a partir de su convivencia con los investigados, participando, dos días por semana, en las actividades de clase de los distintos grupos de la escuela y en el comedor con los maestros.

Las conversaciones informales con padres, docentes y alumnado eran frecuentes en los momentos de observación a través de preguntas del tipo ‘‘cómo ves el ambiente de la escuela?’, ‘¿estás satisfecho/a con tu trabajo en este centro?’, ‘¿te gusta estudiar aquí?’. Estas conversaciones de carácter amistoso permitieron profundizar, contrastar y obtener más detalles (Spradley, 1979) sobre aspectos que iban emergiendo de la investigación (Lincoln y Guba, 1985). De este modo, la permanencia en el campo y la participación del investigador en las actividades cotidianas del centro facilitaron los momentos y la creación de un ambiente de confianza propicio para la obtención y el contraste de datos.

Los tres maestros-tutores y el director del CRA, además de aportar informaciones muy relevantes, facilitaron al investigador el acceso al campo y, con ello, las relaciones con el resto de investigados. Por otra parte, fueron especialmente provechosas las conversaciones con el alumnado de $5^{\circ}$ y $6^{\circ}$ de Educación Primaria. 


\section{N V E S T I G A C I Ó N}

Los primeros contactos con el colectivo de padres y madres fueron con aquellos que tenían una relación más próxima con la escuela. Gracias al ambiente de confianza generado con el transcurso de los días, la participación del investigador en las actividades de convivencia desarrolladas al final del estudio de campo fue muy fructífera. En este sentido, son de destacar las aportaciones de tres madres. Dos de ellas son hermanas nacidas en Pueblogrande y la tercera reside en una población cercana (fue la organizadora de una de las convivencias).

Los documentos del centro, tanto institucionales (Programación General Anual, Memoria del curso y Proyecto de Dirección) como los generados en el día a día, en su condición de productos creados por la comunidad investigada (revista del CRA, páginas web y de Facebook del centro), aportaron datos de gran utilidad (Hammersley y Atkinson, 2007). Todos ellos fueron de gran interés dado que tienen un carácter primario. Son creados por los participantes y resultan de una acción premeditada y reflexionada por su parte.

El cuaderno de notas y la grabadora del teléfono móvil fueron los instrumentos de registro (Hernández y Sancho, 2020). La grabadora fue un instrumento de uso cotidiano con el que todos los participantes estaban familiarizados, sin alterar el ambiente de naturalidad en el proceso de recogida de la información. También se utilizó un cuaderno de diario digital para transcribir las anotaciones, las grabaciones y las impresiones personales del investigador. En las notas tomadas se apuntaba lo observado y lo oído en la forma más exacta posible y luego se trabajaba recordando lo que se había visto pensando sobre ello y en relación con otros estudios. Al final del día se trataba de considerar qué era lo aprendido ese día.

\subsection{Análisis de la información}

El análisis de la información recogida permitió considerar nuevas ideas, a veces distintas a las representaciones existentes sobre los colegios rurales agrupados. La lectura repetida de las notas ayudaba a entresacar las ideas más importantes procedentes de los materiales recogidos y permitían estructurar la información en categorías y elaborar argumentos.

Los referentes analíticos aplicados fueron los principios de la Grounded Theory (Glaser y Strauss, 1967) en su variante constructivista (Charmaz, 2006). Este método ha facilitado la organización y análisis de la información de forma rigurosa. La codificación de los datos surge de los núcleos que fluyen a partir de la lectura de la información recogida de distintas fuentes. Su relectura y comparación de datos y núcleos ha permitido afinar un sistema de categorías y subcategorías interrelacionadas (Charmaz, 2006). Este sistema de interrelaciones, dependencias y vínculos conforma el andamiaje del texto que refleja los resultados, su discusión y las conclusiones surgidas de ello. Como instrumento de apoyo se emplea el software Atlas.ti. Su uso se ha ajustado a las características de la investigación y sus potencialidades, teniendo en cuenta tanto el rigor que se tiene que seguir en el proceso como la creatividad y flexibilidad asociadas a esta metodología. Las categorías se presentan a continuación. 


\section{N V E S T I G A C I Ó N}

Tabla. 1. Categorías.

\begin{tabular}{|c|c|c|c|c|}
\hline \multicolumn{5}{|c|}{ Valor de la escuela local } \\
\hline $\begin{array}{l}\text { Voces de las } \\
\text { familias }\end{array}$ & $\begin{array}{l}\text { Viven en el } \\
\text { pueblo }\end{array}$ & \multirow{2}{*}{$\begin{array}{l}\text { Valoran el } \\
\text { pueblo }\end{array}$} & $\begin{array}{c}\text { Familiaridad/Cordialidad } \\
\text { entre las familias. }\end{array}$ & $\begin{array}{c}\text { Negando la participación con } \\
\text { las familias de escuelas de } \\
\text { otras localidades }\end{array}$ \\
\hline $\begin{array}{l}\text { Voces del } \\
\text { alumnado }\end{array}$ & $\begin{array}{c}\text { En clases de } \\
\text { distintas edades }\end{array}$ & & Lazos de unión & $\begin{array}{c}\text { Ausencia de participación } \\
\text { con el alumnado de escuelas } \\
\text { de otras localidades }\end{array}$ \\
\hline \multirow[t]{2}{*}{$\begin{array}{l}\text { Voces del } \\
\text { profesorado }\end{array}$} & \multirow[t]{2}{*}{$\begin{array}{l}\text { Residen fuera } \\
\text { del pueblo }\end{array}$} & \multirow[t]{2}{*}{$\begin{array}{l}\text { Satisfacción en } \\
\text { la escuela rural }\end{array}$} & $\begin{array}{l}\text { Valoran las relaciones } \\
\text { fáciles con familias }\end{array}$ & \multirow{2}{*}{$\begin{array}{c}\text { Silencios en torno a la } \\
\text { competencia entre escuelas } \\
\text { por mantenerse abiertas }\end{array}$} \\
\hline & & & $\begin{array}{c}\text { Compromiso con el } \\
\text { entornoRefuerzan } \\
\text { conexión entre las distintas } \\
\text { escuelas del CRA }\end{array}$ & \\
\hline
\end{tabular}

Fuente: Elaboración propia

\subsection{Validez}

La información recogida en las 110 horas de trabajo de campo llevadas a cabo durante nueve meses se contrastó desde múltiples perspectivas. En primer lugar, mediante la comparación entre las informaciones aportadas por los participantes y entre estas y las observaciones del investigador reflejadas en el cuaderno de campo (Walford, 2018). A continuación, trasladándolas de nuevo a los participantes (Lincoln y Guba, 1985), preguntándoles si los temas extraídos representaban la realidad interpretada. Así mismo, con la otra investigadora y con otros colegas familiarizados con el proceso de investigación se procedió a la revisión de la información analizada.

Todo esto contribuyó a focalizar la investigación en aquellos aspectos que se consideraban más importantes. Asimismo, se generó una visión del escenario que fue presentada a los participantes a través de informes para su debate y consideración como reflejo de su realidad investigada, y cuyas respuestas se añadieron de nuevo a la información recogida.

De este modo, las informaciones recogidas con la presencia continuada en el campo, la interacción con los participantes, así como la revisión y contraste señalados, junto con los informes devueltos en la última fase reforzaron el proceso de triangulación y fueron importantes para generar verificación y validez a la propia investigación (Creswell, 2007). 


\section{N V E S T I G A C I Ó N}

\section{RESULTADOS}

De acuerdo con el objetivo de identificar y comprender las experiencias e interpretaciones de inclusión o exclusión de familias, docentes y alumnado, en relación con su escuela y con el colegio rural agrupado, los resultados muestran diferencias dependiendo de cómo ocurren las interacciones en el espacio y los lugares. Sin perder de vista que se trata de un estudio etnográfico, los resultados se presentan desde la relación y contraste con las investigaciones revisadas.

Las voces de las familias y del alumnado refuerzan el valor de las relaciones en y con la escuela de su localidad y desde su historia en el lugar frente a las relaciones con los miembros de otras escuelas del CRA en un contexto de mercado que amenaza con el cierre de escuelas.

\subsection{Las familias y el alumnado refuerzan las relaciones en el espacio de su escuela}

Los resultados muestran el gran valor que la localidad, la experiencia vivida en ella y la familiaridad tienen para las familias y el alumnado. Este valor ha sido una constante en las observaciones y en las entrevistas informales con las familias con el alumnado en y fuera de la escuela. La cordialidad entre las familias, la satisfacción de los docentes y las buenas relaciones entre el alumnado de distintas edades son valores y formas de inclusión que se construyen a partir de la interacción social que tiene lugar en la localidad (Corbett, 2015; Massey, 2012).

La cordialidad entre las familias que llevan a sus hijos a la escuela de Pueblogrande es una realidad. Todas, excepto una, residen allí y la mayoría tienen sus raíces en la propia localidad. Las relaciones entre los residentes nacidos en la localidad y los que son oriundos de otros lugares se desarrollan en el sentido señalado por Massey (2012). Una madre comentaba que 'aunque llevo veinte años aquí, me siguen considerando una forastera' (madre1). Sin embargo, e independientemente de que unas familias tengan más relación con otras, todas se unen para implicarse en su escuela.

Las familias de la escuela nos reunimos y seleccionamos actividades para los pequeños [hasta $2^{\circ}$ ] y para los mayores (madre1).

El valor de la localidad y de las relaciones que se generan en esta posibilita el desarrollo del capital social. Las redes en la localidad generan mecanismos de cooperación y facilitan tanto el desarrollo individual como el de la propia comunidad (Putnam, 2002). Sin embargo, estas relaciones no parecen extenderse entre las familias de distintas localidades del CRA. Por ejemplo, cuando hay convivencias o celebraciones del CRA, las familias de cada localidad se juntan entre sí.

Los maestros dicen que nos mezclemos, ¿se mezclan ellos con los padres? Los maestros están con los maestros. Es normal que en una celebración uno esté con la gente que ya conoce y con la que tiene relación (madre2).

Las relaciones entre los miembros de la misma localidad se refuerzan cuando la elección de escuela constituye una amenaza para su existencia. Los intereses entre los miembros de la es- 


\section{N V E S T I G A C I Ó N}

cuela estudiada son contrapuestos a los de otras escuelas del CRA y aquí está presente la lucha porque su escuela no sea cerrada. Las amenazas de cierre de las escuelas más pequeñas ante las fuerzas del mercado son un hecho. 'Las tensiones por el cierre de las escuelas pequeñas están presentes entre las familias' señala un profesor.

Hoy hay una reunión en Pueblogrande promovida por los padres de Pueblonuevo para hablar sobre la jornada continua y no hay acuerdo entre las familias de las distintas localidades. 'Esto puede llevar al cierre de nuestra escuela' señala una madre de otra escuela (cuaderno de notas).

El valor de las relaciones entre el alumnado también está presente en la escuela. El hecho de que sean pocos crea lazos de unión muy fuertes entre ellos (Raggl, 2015). Esto muestra que las relaciones espaciales son importantes y se construyen en los espacios según las experiencias vividas (Massey, 2012).

Claudia coge de la mano a Darío para que no se escape (es muy movido). Los grandes ya saben que hay que controlarlo. Por otra parte, Darío idolatra a Zoilo y éste está también mucho con él. Pablo y Juan también lo controlan. Durante todo el desarrollo de la actividad los grandes cuidan de los pequeños (cuaderno de notas).

Estas relaciones se refuerzan entre los niños de la misma localidad. A pesar de los esfuerzos de los docentes, cuando se juntan en excursiones o convivencias con niños de otras localidades no tienen lugar, 'prácticamente solo se relacionan entre ellos' (cuaderno de notas).

Las valoraciones que hace el alumnado de Pueblogrande con respecto a su marcha al instituto no son menos importantes. Los mayores ( $5^{\circ}$ y $6^{\circ}$ de primaria) no muestran ningún interés en ir al instituto. Vanesa, una alumna de $6^{\circ}$, comenta: 'no entiendo para qué tengo que ir allí [al instituto] si aquí estoy bien'.

Los maestros y las maestras reconocen el valor local de la escuela como un lugar de trabajo ideal (Raggl, 2015) que facilita las relaciones con las familias y con la comunidad (Kalaoja y Pietarinen, 2009).

Yo soy nacido en la zona y he decidido continuar residiendo aquí. Esto me permite mantener una mayor relación con las familias de los alumnos y con los residentes en la localidad (Director).

El compromiso del profesorado con el lugar y con la comunidad lleva a los y las docentes a promover y reconocer la participación de familias y miembros de la comunidad en el desarrollo de actividades (Vigo y Soriano, 2015) de la escuela.

/.../ Como actividad del proyecto, el viernes por la tarde vendrán los padres al aula a explicar distintos objetos antiguos que tengan en su casa /.../ (maestra1).

/.../ En muchas ocasiones echamos mano de vosotros [abuelos y familias]. Por eso nos gusta tener un día especial de agradecimiento desde la escuela para vosotros. Primero porque estáis muy cercanos a 


\section{N V E S T I G A C I Ó N}

la escuela porque la escuela la sentís también vosotros como parte vuestra /.../ (Saludo del director el día de la convivencia de homenaje a los abuelos/as).

El sentido de pertenencia está muy presente entre las familias y el alumnado en la escuela de Pueblogrande. Estos muestran la importante relación de inclusión existente entre los miembros de la localidad vinculada a su espacio y a las condiciones de vida que experimentan. Todos ellos tienen una estrecha conexión con el espacio y eso configura las relaciones en la comunidad (Massey, 2012).

\subsection{Los docentes reconocen cada escuela y refuerzan el valor del Colegio Rural Agrupado}

El profesorado reconoce y refuerza los valores y los intereses de cada escuela. Sin embargo, de acuerdo con las condiciones de su profesión, sus intereses representan a las autoridades educativas (Åberg-Bengtsson, 2009; Grande, 1994) y los intereses del CRA como institución. Las interacciones que tienen lugar en el marco del CRA muestran los esfuerzos del profesorado por mantener y reforzar la estructura y el significado institucional del CRA, a pesar de las situaciones particulares que resultan de un contexto de mercado que amenaza el cierre de las escuelas pequeñas. La voluntad del director y los docentes por conectar las escuelas es la prioridad en el CRA.

El director, máximo representante institucional de todas las escuelas y responsable en gran medida del enfoque que se da a las relaciones del centro con las distintas comunidades educativas, trabaja en el CRA desde su creación. Durante ocho años fue jefe de estudios y desde hace doce años es director. Es maestro especialista y por tanto itinerante, necesariamente, en las cuatro escuelas todas las semanas. Reside en una localidad próxima pero cuya escuela no está integrada en el CRA. Su estabilidad en el centro, el hecho de ser nacido y residente en el territorio y su deseo de abrir las escuelas al entorno le convierten en un referente social (Bagley y Hillyard, 2015; Morales, 2016). 'Su relación con la comunidad va más allá de su condición de director. Es una institución' (maestra 1). Su actividad se integra en la vida social del territorio (Bhengu y Myende, 2015), llegando incluso a localidades cercanas ajenas al CRA.

Otro día fuimos a cantar a la residencia de Pueblomuygrande, pero eso fue a las ocho de la tarde, que eso no lo hace nadie. Allí estaban todos los críos del CRA vestidos de blanco... (maestra1).

La Dirección del CRA trata de generar vínculos entre las distintas comunidades.

/... / Hace años que se organizó un sistema institucionalizado de participación en la gestión del centro que diese voz a ayuntamientos y padres de todas las localidades junto con los docentes. Se consideraba que la configuración legal del Consejo Escolar tenía sus limitaciones si se quería hacer de él un órgano realmente participativo y operativo que tuviera en cuenta la realidad de las escuelas. Es la Comisión de Convivencia. /.../

/.../ Estamos el equipo directivo, los coordinadores de los equipos didácticos, la orientadora y un padre de cada pueblo más un representante de ayuntamientos de cada localidad. /.../. Sobre todo, lo que hacemos es coordinarnos para el tema de las convivencias, las excursiones, cuando tienen que colaborar padres en la escuela... (director). 


\section{N V E S T I G A C I Ó N}

La organización de actividades de convivencia para todo el alumnado del CRA; la potenciación de actividades en común, como son la revista escolar o el blog del Centro; y el trabajo con sistemas telemáticos, son estrategias que se proponen para cubrir la necesidad de socialización que tienen los alumnos de las distintas localidades. (Proyecto de Dirección).

Los docentes intentan promover las relaciones entre el alumnado y familias de las distintas escuelas, del CRA para atenuar las situaciones de soledad del alumnado y cohesionarlo como colectivo silenciando en ocasiones los condicionantes que amenazan con el cierre de la escuela. Los y las docentes conocen las tensiones que experimentan las familias de la localidad para captar alumnado ante el posible cierre de su escuela. Sin embargo, cuando se pregunta sobre la situación, señalan que 'esa es una cuestión de las familias'.

\section{DISCUSIÓN Y CONCLUSIONES}

Los resultados y la reflexión teórica expuestos en este artículo muestran consideraciones de interés para el funcionamiento de las instituciones educativas. Estas consideraciones se destacan en un contexto en el que la educación, de acuerdo con la Agenda 2030, se reconoce como un bien común universal y como base para mejorar la calidad de vida de las personas y para el desarrollo sostenible ante la falta de escuelas y de condiciones que aseguren la inclusión y la equidad a niños y niñas de zonas rurales.

La atención a las particularidades de los contextos y lugares es un aspecto importante. Además, no todas las escuelas son iguales, sean rurales o urbanas. Las voces de familias, alumnado y docentes de las escuelas rurales en este estudio subrayan el valor del espacio local y la tensión que viven y experimentan en un contexto de mercantilización de las escuelas. Más específicamente, en la escuela estudiada, se manifiestan diferencias entre las acciones y experiencias de familias y las de los docentes. De una parte, el compromiso de familias y el alumnado con su escuela local es un hecho. De otra parte, solo los maestros experimentan un compromiso con respecto al CRA. Así, el artículo muestra el sentido de las relaciones espaciales y las limitaciones que presenta la aplicación de un modelo de escuela rural, con 'largos pasillos' que sigue ignorando las voces de los implicados, cuando a la vez se habla de inclusión y de sostenibilidad (UNESCO, 2018).

Las familias y el alumnado refieren el valor de las relaciones con el lugar concreto en el que viven y en el que ocurren las interacciones (Bagley y Hillyard, 2015; Johansson, 2017). Esa representación indica una clara relación espacial de inclusión con su localidad (Beach, From, Johansson y Öhrn, 2018; Massey, 2012) distinta a la que experimentan los docentes, más centrados en los objetivos de su institución y por tanto más próxima a la administración educativa que representan (Åberg-Bengtsson, 2009; Grande, 1994). Los docentes, como representantes institucionales y sin residir en la localidad de la escuela, en su mayor parte, tratan de reforzar las relaciones entre las escuelas ubicadas en distintos lugares. En este sentido, la comprensión del espacio y del lugar como extensión de las interacciones entre sus miembros parece ser importante para generar vínculos entre las familias y el alumnado con su escuela de una parte y entre el profesorado de otra (Putnam, 2002). Las implicaciones para la formación del profesorado en las instituciones de educación superior son relevantes atendiendo a una dimensión reflexiva y crítica. 


\section{N V E S T I G A C I Ó N}

Los resultados de la investigación también sugieren que las interacciones son importantes para entender las relaciones de poder entre la escuela y la comunidad (Johansson, 2017), afectando la comunicación entre ambas. Según Massey (2012), la comprensión del espacio como socialmente construido y las relaciones sociales como construcciones espaciales hacen de la comunicación un punto crítico. Así, las relaciones entre familias y alumnado en torno a la institución CRA, pese a compartir proyectos y objetivos, contrastan con sus interacciones en la escuela de la localidad.

La negación de las relaciones entre las familias del CRA ocurre ante las condiciones de un contexto neoliberal guiado por valores de mercado. Los profesores, bajo las orientaciones de la administración educativa y del modelo CRA, tratan de articular relaciones y vivencias compartidas entre familias y alumnado, pero éstas no son siempre aceptadas por las familias. Las familias reaccionan con resistencia ante estas relaciones entre las distintas localidades promovidas por los profesores de acuerdo con las normas dadas desde el CRA. Las amenazas experimentadas por las familias respecto a perder su escuela y su espacio están presentes.

Las identidades rurales en educación son factores importantes a considerar para entender las distintas perspectivas de las familias y el alumnado desde las decisiones políticas y para entender al profesorado (Llevot y Garreta, 2008). Siguiendo a Massey (2012) las relaciones de exclusión y de poder están presentes. El valor local en una escuela se presenta como una realidad frente a la estructura administrativa de un CRA que sigue funcionando con criterios de escuela urbana y de mercado. Las familias perciben que las experiencias de la población que vive en áreas rurales no se tienen en cuenta en las políticas educativas. De acuerdo con Soja (2014), es importante reconocer la situación de desigualdad y abstracción de la escuela rural cuando se siguen aplicando modelos de escuela urbana estandarizada, aun sin ignorar que las escuelas rurales también son diferentes. Los resultados muestran la necesidad de diferenciar el funcionamiento y las necesidades de las distintas escuelas rurales (Vigo y Soriano, 2015; Vigo y Beach, 2018; Massey, 2012; Ortega, 1995).

A la vista de los resultados señalados, cuando se reconoce la educación como base para mejorar la calidad de vida de las personas y para el desarrollo sostenible que aseguren la inclusión y la equidad en las zonas rurales, resulta obligado que las administraciones educativas reconsideren las políticas y prácticas educativas en este sentido. Dentro de la escuela, cuando se habla de desarrollo sostenible, igualmente es de obligada consideración reconocer las voces y las necesidades que experimentan familias y alumnado en los espacios que se encuentran en desventaja desde el punto de vista del acceso a la escuela de la localidad. Esto significa emprender acciones, desde los gobiernos, las administraciones educativas y desde las instituciones de formación de profesorado, que faciliten y promuevan el verdadero desarrollo. Garantizar la inclusión y la equidad así como el desarrollo sostenible requiere en primer lugar, escuchar, reconocer y valorar las voces de quienes habitan los espacios rurales en la actualidad. En segundo lugar, es importante articular acciones que garanticen el desarrollo.

De acuerdo con el objetivo 4 de la Agenda 2030, la educación para el desarrollo sostenible y la adopción de estilos de vida sostenibles, así como la valoración de la diversidad cultural y de la contribución de la cultura al desarrollo sostenible contribuyen a la inclusión. No obstante, en un 


\section{N V E S T I G A C I Ó N}

contexto en el que coexisten valores de mercado con valores de inclusión y desarrollo sostenible, el conflicto está presente. Reconocer el discurso de inclusión y desarrollo sostenible no es garante de cambio si no se asumen y articulan acciones que realmente lo posibiliten. En este artículo se destaca el sentido de escuchar, reconocer y contribuir al desarrollo sostenible de las zonas rurales atendiendo a los valores ya existentes en la escuela rural frente a la 'nueva mercantilización’ de las escuelas rurales (Farrugia, 2020).

Actualmente en España, aunque el esfuerzo de la administración por mantener abiertas escuelas pequeñas ha sido importante (Vigo y Soriano, en prensa), no se puede hablar de coherencia en las políticas educativas de zonas rurales. Las voces de las familias, alumnado y docentes confirman esta división y señalan diferentes relaciones de poder con respecto a las escuelas rurales. Esto indica la necesidad de una discusión crítica y de una política que contribuya a reconocer las necesidades que surgen en las distintas escuelas así como a buscar respuestas que garanticen su existencia. La perspectiva inclusiva y de desarrollo sostenible exige insistir en la investigación a propósito de las dinámicas que se dan en las distintas escuelas pequeñas y en los CRAs y en la detección de prácticas que respondan a las necesidades de las localidades. Los estudios de investigación en profundidad aportan conocimiento a la comunidad investigadora y a las realidades investigadas.

\section{REFERENCIAS}

Åberg-Bengtsson, L. (2009). The Smaller the Better? A Review of Research on Small Rural Schools in Sweden. International Journal of Educational Research, 48(2), 100-108. http://doi.org/10.1016/j. ijer.2009.02.007

Abós, P., y Lorenzo, J. (2019). El binomio educativo de los colegios rurales agrupados y los centros rurales de innovación educativa: un referente de calidad organizativa y pedagógica para la escuela rural de Teruel (España). Revista Espaço do Currículo, 12(1), 5-24. Doi:10.22478/ufpb.19831579.2019v12n1.41930

Álvarez, C.,yVejo, R. (2017). ¿Cómo se sitúanlas escuelas españolas del medio rural antelainnovación? Un estudio exploratorio mediante entrevistas. Aula Abierta, 45, 25-32. Doi:10.17811/rifie.45.2017.25-32

Autti, O. \& Hyry-Beihammer, E. K. (2014). School closures in rural Finnish communities. Journal of Research in Rural Education, 29(1), 1-17. Recuperado de https://jrre.psu.edu/sites/default/files/2019-08/29-1.pdf

Bagley, C., y Hillyard, S. (2015). School choice in an English village: living, loyalty and leaving. Ethnography and Education, 10(3), 278-292. Doi:10.1080/17457823.2015.1050686

Beach, D. (2017). Whose justice is this! Capitalism, class and education justice and inclusion in the Nordic countries: race, space and class history. Educational Review, 69(5), 620-637. http://doi.org/1 $\underline{0.1080 / 00131911.2017 .1288609}$

Beach, D., From, T., Johansson, M. y Öhrn, E. (2018). Educational and spatial justice in rural and urban areas in three Nordic countries: a meta-ethnographic analysis. Education Inquiry, 9(1) 4-21. Doi:10. $1080 / 20004508.2018 .1430423$

Bhengu, T. T., y Myende, P. E. (2015). Traversing metaphoric boundaries between schools and their communities: An ethnographic study of a rural school. Journal of Social Sciences, 43(3), 227-236. https://doi.org/10.1080/09718923.2015.11893440 


\section{N V E S T I G A C I Ó N}

Boix, R. (2004). La escuela rural: Funcionamiento y necesidades. Las Rozas Madrid: CissPraxis.

Camarero. L. (2017). Por los senderos de la despoblación: notas desde la diversidad social. Documentación Social, 185, 19-35. Recuperado de https://caritas-web.s3.amazonaws.com/mainfiles/uploads/2019/04/DocSocial185-1.pdf

Charmaz, K. (2006). Constructing grounded theory: A practical guide through qualitative analysis. London: Sage Publications.

Corbett M. (2015). Towards a rural sociological imagination: ethnography and schooling in mobile modernity. Ethnography and Education, 10(3), 263-277. https://doi.org/10.1080/17457823.2015.1050 $\underline{685}$

Corchón, E., Raso, F., y Hinojo, M. A. (2013). Análisis histórico-legislativo de la organización de la escuela rural española en el período 1857-2012. Enseñanza y Teaching: Revista Interuniversitaria de Didáctica, 31(1), 147-179. Recuperado de https://revistas.usal.es/index.php/0212-5374/article/ view/11609

Creswell, J. W. (2007). Qualitative inquiry and research design: Choosing among five approaches. Thousand Oaks, London, New Delhi: Sage Publications.

Domingo, V. (2013). Origen y evolución del modelo CRA-Colegio Rural Agrupado-. Situación en Aragón y estudio de caso de la realidad turolense (Tesis doctoral). Valencia: Universidad de Valencia.

Farrugia, D. (2020). Class, place and mobility beyond the global city: stigmatisation and the cosmopolitanisation of the local. Journal of Youth Studies, 23(2), 237-251. Doi:10.1080/13676261.20 19.1596236

García, F. J., y Pozuelos, F. J. (2017). El currículum integrado: los proyectos de trabajo como propuesta global para una escuela rural alternativa. Aula Abierta, 45, 7-14. Doi:10.17811/rifie.45.2017.7-14

Garreta, J. (2017). Familias y escuelas. Discursos y prácticas sobre la participación en la escuela. Madrid: Pirámide.

Giordano, E. A. (2008). School clusters and teacher resource centres. Paris: UNESCO International Institute for Educational Planning (IIEP).

Glaser, B. y Strauss, A. (1967). The discovery of grounded theory. Chicago: Aldine Press.

Grande, M. (1994). La escuela rural en España. De la LGE A la LOGSE. Balance y perspectivas (Tesis Doctoral). Salamanca: Universidad de Salamanca.

Hammersley, M. (2018). What is ethnography? Can it survive? Should it? Ethnography and Education, 13(1), 1-17. Doi:10.1080/17457823.2017.1298458

Hammersley, M., y Atkinson, P. (2007). Ethnography: Principles in practice. London: Routledge.

Hargreaves, L. (2017). Primary education in small rural schools: past, present and future. En R. Maclean (Ed.). Life in Schools and Classrooms (pp. 223-243). Singapore: Springer. Doi:10.1007/978-981-103654-5_14

Hernández, F., y Sancho, J. M. (2020). Writing and Managing Multimodal Field Notes. En G. W. Noblit (Ed.). The Oxford Encyclopedia of Qualitative Research Methods in Education. New York: Oxford University Press. https://doi.org/10.1093/acref/9780190643751.001.0001 


\section{N V E S T I G A C I Ó N}

Hernández, J.M. (2000). La escuela rural en la España del siglo XX. Revista de Educación, Número extraordinario, 113-136. Recuperado de https://sede.educacion.gob.es/publiventa/descarga.action?f codigo_agc $=8522 \_19$

Jachyra, P., Atkinson, M., yWashiya,Y. (2015). 'Who are you, and what are you doing here': methodological considerations in ethnographic Health and Physical Education research. Ethnography and education, 10(2), 242-261. Doi:10.1080/17457823.2015.1018290

Jeffrey, B. y Troman. G. (2004) Time for ethnography. British Educational. Research Journal, 30(4), 535 548. https://doi.org/10.1080/0141192042000237220

Johansson, M. (2017). "Yes, the power is in the town": An ethnographic study of student participation in a rural Swedish secondary school. Australian and International Journal of Rural Education, 27(2), 6176. Recuperado de https://journal.spera.asn.au/index.php/AIJRE/article/view/118/120

Kalaoja, E., y Pietarinen, J. (2009). Small rural primary schools in Finland: A pedagogically valuable part of the school network. International Journal of Educational Research, 48(2), 109-116. Doi:10.1016/j. ijer.2009.02.003

Llevot, N., y Garreta, J. (Eds.). (2008). Escuela ruraly sociedad. Lleida: Universitat de Lleida.

Lorenzo, J., Domingo, V., Nolasco, A. y Abós, P. (2019). Analysis of educational leadership at rural earlychildhood and primary schools: a case study in Teruel (Aragon, Spain). International Journal of Leadership in Education. Doi:10.1080/13603124.2019.1657590

Martínez, J., y Bustos, A. (2011). Globalización, nuevas ruralidades y escuelas. Profesorado. Revista de Currículum y Formación de Profesorado, 15(2), 3-12. Recuperado de https://recyt.fecyt.es/index.php/ profesorado/article/view/43399/25273

Massey, D. (2012). Space, place and gender. Cambridge: Polity Press.

Morales, N. (2016). Rural Schools in Spain. Past, present and future: a sociological framework. En M. Gather, A., Lüttmerding, J., Berding, y G. Villarroel (eds). Education, Local Economy and Job Opportunities in Rural Areas in the context of demographic change (pp. 3-12). Erfurt: Institut Verkehr und Raum.

Öhrn, E. y Beach, E. (2019). Young people's life and schooling in rural areas. London: The Tufnell Press.

Ortega, M. A. (1995). La parienta pobre. (Significantey significados de la Escuela Rural). Madrid: CIDE.

Poirey, J. (2006). Réussite scolaire au collège et implication des acteurs: L'action au carrefour des territoires et des réseaux. Alpe, Y., Champollion, P. y Poirey, J. (Coords.). L'enseignement scolaire en milieu rural et montagnard. Le devenir des élèves en fin de collège: Parcours et projets, 4, (121-126). Besançon: Presses Universitaires de Franche-Comté.

Ponce de León, A., Bravo, E., y Torroba, T. (2000). Los colegios rurales agrupados, primer paso al mundo docente. Contextos educativos: Revista de educación, 3, 315-348. Doi:10.18172/con.428

Putnam, R. (2002). Solo en la bolera: Colapso y resurgimiento de la comunidad norteamericana. Barcelona: Galaxia Gutemberg.

Raggl, A. (2015). Teaching and learning in small rural primary schools in Austria and Switzerland. Opportunities and challenges from teachers' and students' perspectives. International Journal of Educational Research, 74, 127-135. https://doi.org/10.1016/j.ijer.2015.09.007

Raggl, A. (2018). Fieldnote issues, collegial support and collaborative analysis. En B. Jeffrey y L. Russell (Eds.), Ethnographic writing (191-200). Gloucestershire, UK: E\&E Publishing. 


\section{N V E S T I G A C I Ó N}

Sales, A., Moliner, O. y Lozano, J. (2017). Strategies to link schools to their territories. A survey study. Procedia - Social and Behavioral Sciences, 237, 692-697. https://doi.org/10.1016/j.sbspro.2017.02.044

Santamaría, R. (2012). Inspección de educación y escuela rural. Contra el mito del bajo rendimiento de la escuela rural. Visión histórica 1972-2012. Revista de la Asociación de Inspectores de Educación de España, 17, 1-29. Recuperado de https://avances.adide.org/index.php/ase/article/view/521

Soja, E. W. (2014). En busca de la justicia espacial. Valencia: Tirant Humanidades.

Spradley, J. P. (1979). The ethnographic interview. New York: Holt, Rinehart y Winston.

Tapia, L. y Castro, P. (2014) Experiencia educativa. Educar desde un CRA. Revista Tendencias pedagógicas, 24, 415-428.

Torres, I., y Simovska, V. (2017). Community participation in rural Ecuador's school feeding programme: A health promoting school perspective. Health Education, 117(2), 176-192. https://doi.org/10.1108/ HE-02-2016-0009

UNESCO (2018). Informe de seguimiento de la educación en el mundo de 2020. Nota conceptual. París: UNESCO. Recuperado de https://unesdoc.unesco.org/ark:/48223/pf0000265329_spa

Vázquez, R. (2016). Las escuelas públicas rurales: entre el bien común y la exclusión. Revista Interuniversitaria de Formación del Profesorado, 85, 67-79.

Vigo, B., y Soriano, J. (2015). Family involvement in creative teaching practices for all in small rural schools. Ethnography and Education, 10(3), 325-339.

Vigo, B., y Beach, D. (2018). Significados de la escuela rural desde la investigación. Representaciones compartidas entre España y Suecia en la segunda parte del siglo xx y primeros años del siglo XXI. En Educació i desenvolupament rural als segles xix-xx-xxi (pp. 225-236). Lleida: Universitat de Lleida.

Vigo, B., y Soriano, J. (En prensa). Development and research of the rural school situation in Spain. In C Gristy, L. Hargreaves, y S.R. Kučerová (Coords.) Educational research and schooling in rural Europe: An engagement with changing patterns of education, space and place. IAP Publishing.

Walford, G. (2018). Recognisable continuity. A defence of multiple methods. En D. Beach, C. Bagley y S. Marques da Silva (Eds). The Handbook of Ethnography of Education (pp. 17-30). London and New York: Wiley.

Yull, D., Wilson, M., Murray, C., y Parham, L. (2018). Reversing the Dehumanization of Families of Color in Schools: Community-Based Research in a Race-Conscious. Parent Engagement Program. School Community Journal, 28(1), 319-347.

\section{Agradecimientos:}

Artículo vinculado al Proyecto de Investigación 'La atención a la diversidad en los núcleos rurales dispersos de la Comunidad de Aragón (CEDDAR: DT 15 (2009-1)’ y al Proyecto 'Familias y Escuelas. Discursos y prácticas cotidianas sobre la participación de las familias en la educación obligatoria (EDU2012-32657)'. 